\title{
Investigation of Dynamic Dichroism Properties of Disperse Orange 11 Molecules and Azo-Molecules Doped in Poly(Methyl Methacrylate) Thin Films: A Comparison
}

\author{
B. AbBas* and M. Alshikh Khalil \\ Atomic Energy Commission, P.O. Box 6091, Damascus, Syria \\ (Received January 20, 2010; in final form Feruary 26, 2010)
}

\begin{abstract}
We investigate the dichroism characteristics of poly (methyl methacrylate) PMMA thin films doped with DO11, DO3, DSR13 and DSR1 dye molecules, which change under illumination with visible light. Our data show that photoisomerisation, phototautomerization reactions and light-induced polar orientation are dependent on the molecular structure of the polymer. Polar structure and molecular size, as well as the chemical structure of PMMA host, are the dominant parameters that determine both the extent and speed of dichroism buildup and relaxation processes. Two forms of DO11 molecules (keto and enol forms), and two other forms of azo-based dyes (trans and cis forms) are responsible for the interaction with irradiating laser light and forming an anisotropic structure inside the PMMA/dye thin films.
\end{abstract}

PACS numbers: 33.55.+b, 78.20.e, 78.66.Qn

\section{Introduction}

The importance of organic and polymeric nonlinear optical (NLO) materials for applications in nonlinear optics is widely recognized, and there has been intensive research on these materials in the past decade [1-2]. Recently, photo-induced optical anisotropy in polymer/organic dye systems has been the subject of intensive investigations [3-4]. These materials are promising in the field of modern optics for optical recording, optically-controlled optical elements, optical switches, and so on. Numerous studies on azo dyes incorporated in polymeric systems have been reported in the past two decades [3-5]. Anthraquinone dyes have also been under extensive research as well, since they have interesting optical properties. Of special interest, nonlinear optical properties of Disperse Orange 11 (DO11) dye have been explored in the field of solid dye lasers owing to its recognized optically pumped lasing action at the attractive wavelength of $650 \mathrm{~nm}$. The dye was incorporated into poly(methyl methacrylate) (PMMA) rods, and amplified spontaneous emission was studied under second-harmonic Nd:YAG laser excitation in a transverse pumping configuration. Gain and conversion efficiency were found to be comparable with those for other laser dyes [6-7].

Measurements of the absorption of linearly polarised light by an ensemble of oriented molecules form also a

* corresponding author; e-mail: scientific@aec.org.sy very important method available for studying the structure and physical behaviour of nonlinear optical guesthost systems. In other words, dichroism is a measure of the orientation of the nonlinear optical dye molecules in the host polymer [8]. This molecular orientation under the influence of the polarized irradiation light leads to a non-central-symmetric alignment of the dye molecules. Nevertheless, although dichroic properties of azobenzene-besed dyes/PMMA have been heavily studied in the past, dichroism studies on DO11 molecules are rather rare in literature. To our knowledge, linear dichroism of DO11/PMMA thin films has never been studied before. In this paper, we study photoinduced linear dichroism properties of DO11 molecules that have been incorporated in PMMA polymeric network. Based on the traditional pump-probe technique, the linear dichroic properties of guest-host polymeric thin films were studied, in which DO11, DO3, DSR13, and DSR1 dyes (guest materials) were dispersed in PMMA polymer network (host material). Continuous Argon laser light was used to pump and probe the samples. Photo-induced dichroism was investigated in these samples. A comparison study between the two species of dye (Azo- and anthraquinonebased) is presented as well.

\section{Experimental}

\subsection{Sample preparation}

Poly(methyl methacrylate) (PMMA, MW = 36000) from Acros Organics, and 1-amino-2-methylanthraquinone (DO11, $\approx 95 \%$ dye content), 
N-Ethyl-N-(2-hydroxyethyl)-4-(4-nitrophenylazo)aniline (DSR1, $\approx 95 \%$ dye content), 4-(4-Nitrophenylazo)aniline (DO3, $\approx 90 \%$ dye content $)$, and 2-[[4-[(2-chloro-4-nitrophenyl)azo]phenyl]ethylamino]ethanol (DSR13, $\approx 95 \%$ dye content) are the starting materials for preparing the guest-host polymeric samples. All dyes were purchased from Aldrich, and were used as received. The film samples were prepared as follows: PMMA $(2 \mathrm{~g})$ was dissolved in $20 \mathrm{ml}$ of Dichloromethane $\left(\mathrm{CH}_{2} \mathrm{Cl}_{2}\right)$ in four separate Erlenmeyer flasks. Clear solutions were obtained. Dyes were added to these clear solutions (equivalent to $5 \%$ of PMMA by weight) so that each solution contains one dye only. The mixtures were, then, stirred for 5-6 h until clear solutions were obtained with dyes fully dissolved. Thin films with thicknesses (measured by the prism coupling technique) of the order of $1-2 \mu \mathrm{m}$ were dip-coated on glass substrates. Directly following the dip-coating procedure, samples were baked in an oven and held at $70^{\circ} \mathrm{C}$ for one hour in order to eliminate the residual solvents.

\subsection{Experimental setup}

Upon pumping of the dye molecules with linearly polarised light, the guest-host polymeric thin films become dichroic. The absorbances parallel $\left(A_{\|}\right)$and perpendicular $\left(A_{\perp}\right)$ to the electric vector of the pump beam become different. However, another laser beam, linearly polarised (probe), is used to measure the dichroism. The absorbance of the probe beam is measured when it is polarised both parallel and perpendicular to the polarisation direction of the pump beam. However, it is very important to use a very weak probe beam with a wavelength very close to the absorption maximum of the dye molecules where any changes in the absorption will be greatest. The absorbances were then calculated from:

$$
A_{\|}=-\log _{10}\left(\frac{I_{\|}}{I_{o}}\right)
$$

and

$$
A_{\perp}=-\log _{10}\left(\frac{I_{\perp}}{I_{o}}\right),
$$

where $I_{O}$ is the intensity of the probe beam reaching the detector when there is no sample, and $I_{\|}$and $I_{\perp}$ are the intensities of the probe beam transmitted through the sample when it is polarised parallel and perpendicular to the pump beam polarization, respectively. The average absorbance, $A_{\mathrm{a}}$, is given by:

$$
A_{\mathrm{a}}=\frac{A_{\|}+2 A_{\perp}}{3} .
$$

This average absorbance represents the isotropic absorption, which does not depend on the medium anisotropy, and it characterises only the relative concentration of trans isomers of the azo-based dyes, or the keto forms of DO11 molecules present in the samples [9]. The dichroic ratio (the order parameter) was computed from the recorded spectra as [10]:

$$
D=\frac{A_{\|}-A_{\perp}}{A_{\|}+2 A_{\perp}} .
$$

In order to find the best suitable absorption wavelength for the samples, a UV-visible spectrophotometer (Photodiode Array Photospectrometer (PDA) Specord S100, from Analytik Jena) was used to record the UV-visible absorption spectra. Figure 1 shows the maximum absorption wavelengths for all prepared guest/host samples. Hence, a light with a wavelength similar, or as close as possible, to the maximum absorption of each sample for both pump and probe beams has to be used in this investigation. The chemical structure of the organic dyes are shown in Table I.

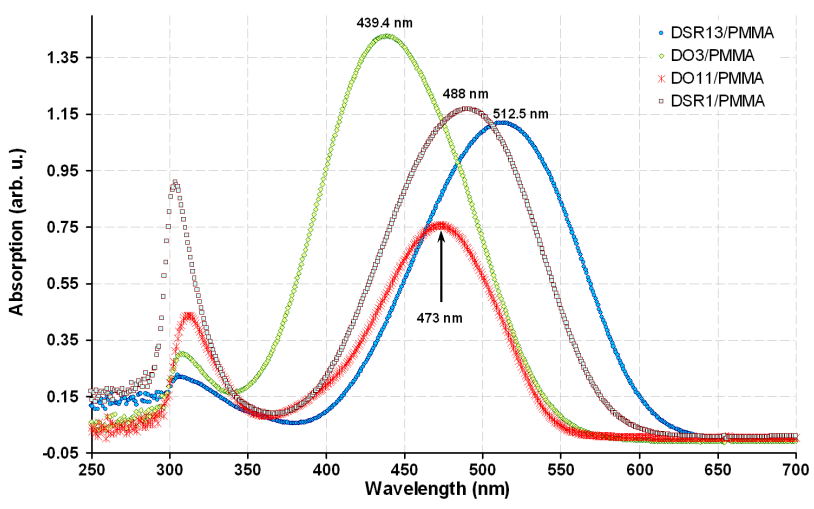

Fig. 1. UV-Visible absorption spectra of dyes used in this work.

The appropriate wavelengths were provided by the multi-line Ar-ion laser (543-MAP-A02, from Melles Griot). Table I shows the wavelengths at which the maximum absorptions of our samples take place, the laser wavelengths that are provided by the laser for each sample, and the chemical structure of the organic dyes. Photo-induced dichroism was measured using the experimental arrangement shown in Fig. 2.

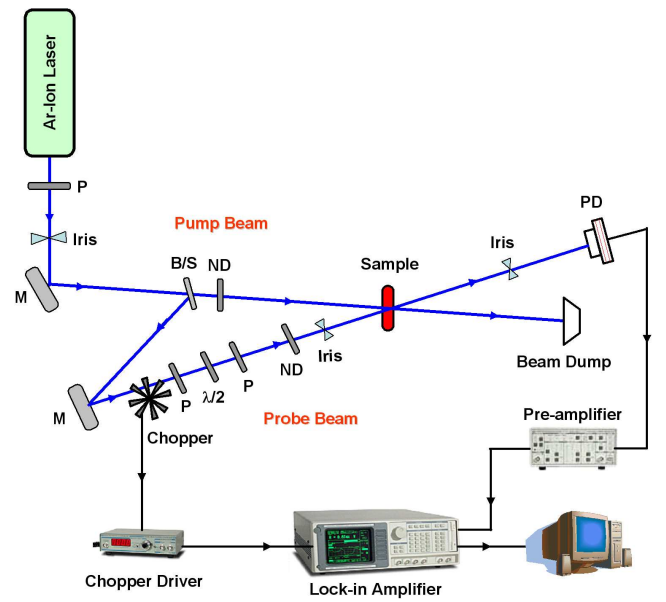

Fig. 2. The experimental setup: P: polariser, ND: neutral density filter, $\mathrm{B} / \mathrm{S}$ : beam splitter, $\lambda / 2$ : half-wave plate, and PD: photodiode. 
TABLE I

The chemical structures of the organic dyes, the maximum absorption is deduced from Fig. 1 and the suitable pumping laser lines are available from the Ar-ion laser.

\begin{tabular}{c|c|c|c}
\hline \hline $\begin{array}{c}\text { Guest/Host } \\
\text { Sample }\end{array}$ & Chemical Structure of the dye & $\begin{array}{c}\text { Maximum } \\
\text { absorption } \\
\text { [nm] }\end{array}$ & $\begin{array}{c}\text { Available } \\
\text { Ar }\end{array}$ \\
line [nm]
\end{tabular}

TABLE II

Summary of absorption and dichroism levels at the photostationary state in all polymeric thin film samples.

\begin{tabular}{c|c|c|c|c|c}
\hline \hline $\begin{array}{c}\text { Guest/Host } \\
\text { System }\end{array}$ & $\begin{array}{c}\text { Used } \\
\text { Wavelength } \\
{[\mathrm{nm}]}\end{array}$ & $\begin{array}{c}\text { Parallel } \\
\text { Absorption } \\
\left(A_{\|}\right)\end{array}$ & $\begin{array}{c}\text { Perpendicular } \\
\text { Absorption } \\
\left(A_{\perp}\right)\end{array}$ & $\begin{array}{c}\text { Average } \\
\text { Absorption } \\
\left(A_{\mathrm{a}}\right)\end{array}$ & $\begin{array}{c}\text { Dichroism } \\
|D|\end{array}$ \\
\hline DO11/PMMA & 472 & 2.012 & 2.396 & 2.1399 & 0.0565 \\
DO3/PMMA & 457 & 1.984 & 2.279 & 2.1805 & 0.0452 \\
DSR13/PMMA & 514 & 0.723 & 1.0825 & 0.9625 & 0.3601 \\
DSR1/PMMA & 488 & 2.5043 & 2.841 & 2.7287 & 0.0411
\end{tabular}

TABLE III

Rise time constants and breadth parameters of guest/host samples.

\begin{tabular}{c|c|c|c|c}
\hline \hline & DO11/PMMA & DO3/PMMA & DSR13/PMMA & DSR1/PMMA \\
\hline$\tau$ [seconds] & 109.031 & 175.77 & 22.55 & 7.52 \\
$\beta$ & 0.63 & 0.095 & 0.455 & 0.213
\end{tabular}

TABLE IV

Relaxation time constants and breadth parameters of guest/host samples.

\begin{tabular}{c|c|c|c|c}
\hline \hline & DO11/PMMA & DO3/PMMA & DSR13/PMMA & DSR1/PMMA \\
\hline$\tau$ [seconds] & 349.45 & 202.49 & 83.95 & 17.24 \\
$\beta$ & 0.175 & 0.158 & 0.243 & 0.215
\end{tabular}

\section{Results}

\subsection{Dichroism}

The pump beam intensity was kept $\approx 16 \mathrm{~mW} / \mathrm{cm}^{2}$ with vertical polarization for all measurements, while the probe beam intensity was $\approx 0.03 \mathrm{~mW} / \mathrm{cm}^{2}$ for both horizontal and vertical polarizations for all measurements. Figure 3 shows a representative experimental result for a $1.5 \mu \mathrm{m}$ thin film of DO3/PMMA. $A_{\|}, A_{\perp}, A_{\mathrm{a}}$, and $D$ 
were calculated according to Eqs. (1)-(4). These calculations were repeated for other systems as well. The explanation of the absorption behaviour illustrated in Fig. 3 is well documented in previous works of many authors, as well as our published work $[9,11]$.

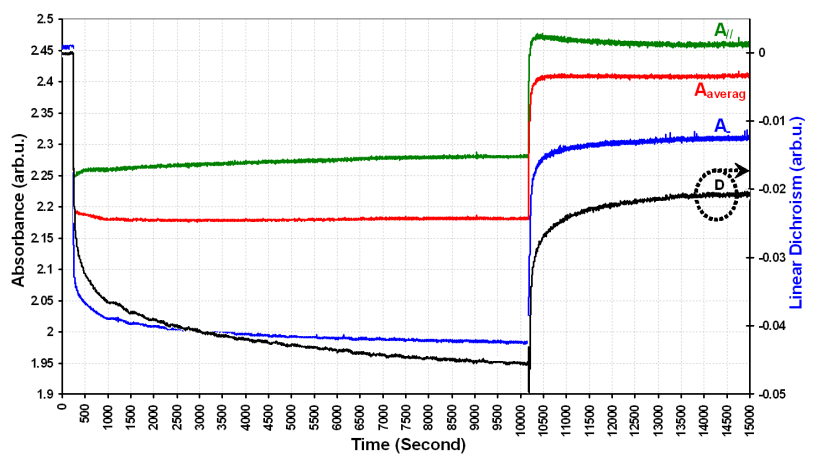

Fig. 3. Absorption and dichroism calculations of a $1.5 \mu \mathrm{m}$ thin film of DO3/PMMA.

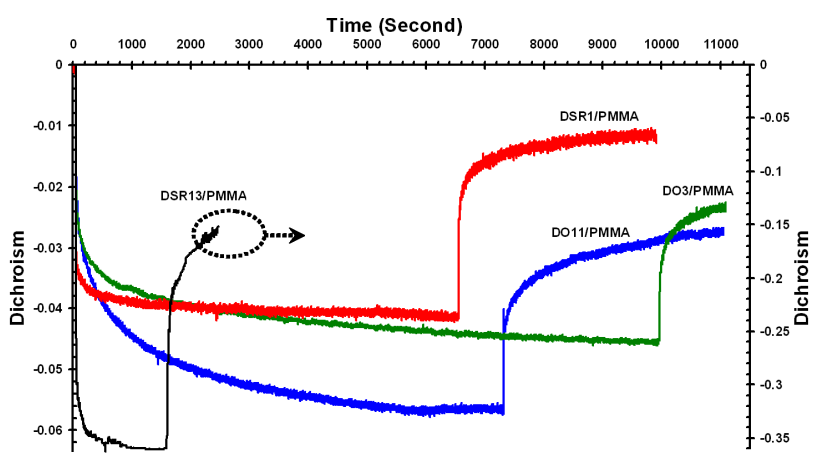

Fig. 4. Dichroism calculations for all guest/host polymeric systems. All sample were $1.5 \mu \mathrm{m}$ thin films.

Figure 4 shows the result of calculation of the induced dichroism in the $1.5 \mu \mathrm{m}$ thin film samples using Eq. (4). The kinetics of the calculated dichroisms emphasize the ability for dye molecules to react with the pump laser light and form a polar order in the sample in a fast fashion. Dichroism values reach a plateau in analogy with the photostationary states of the polymeric systems. However, they also diminish as soon as the pump irradiation is cut off, manifesting the tendency of the polymeric systems towards the isotropic states of the dye molecule distributions within the polymer networks. These observations indicate that these changes are completely reversible, leading to the conclusion that keto $\Leftrightarrow$ enol of DO11 or trans $\Leftrightarrow$ cis of azo-based dyes were occurring in the guest-host polymeric thin films [9]. Table II summerises the dichroism measurements for all samples.

DSR13/PMMA samples showed very high values of dichroism compared to the rest of the guest/host systems. This could be attributed to the chemical structure of the dye, which is smaller than that of other dyes, as well as to its high value of dipole moment (which will be discussed later). This structure allows the isomers of DSR13 molecules to interact with pumping laser light and absorb it in a fast and efficient fashion, which, in turn, facilitates the trans $\Leftrightarrow$ cis photoisomerisation process. This is also manifested in the short pumping time needed for the DSR13/PMMA system to reach the photostationary state, which will be discussed later in this paper.

\subsection{Dichroism rise-up dynamics}

As soon as the pump beam starts to hit the samples, dichroism value for all polymeric systems increases rapidly in the beginning, then it is followed by a slower increase, until it reaches a plateau in analogy with the photostationary states of the polymeric systems. Figure 5 shows the photo-induced dichroism rising phase during the irradiation process. The difference in dichroism extent between those systems does reflect the difference in the molecular mobility of dye molecules in these systems.

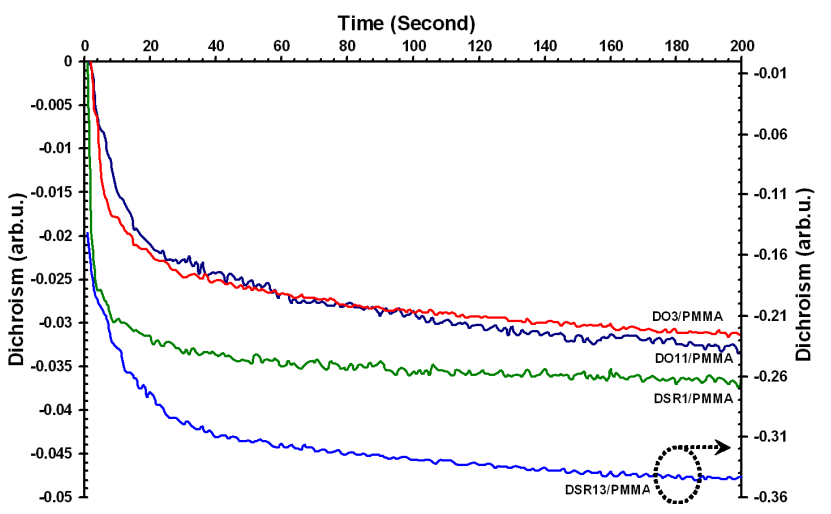

Fig. 5. Dichroism build-up process under the influence of polarized laser irradiation.

The dynamic behaviour of photo-induced dichroism build-up is fitted by a Williams-Watt stretched exponential [12], which describes the rise phenomena, and given as:

$$
D(t)=D_{o} \exp \left(-\left(\frac{t}{\tau}\right)^{\beta}\right) .
$$

where $t$ is the time at which $D(t)$ is measured. $D_{0}$ is the dichroism of the sample prior to sample irradiation, $\tau$ is a characteristic rise time, $\beta(0 \leq \beta \leq 1)$ is a distribution parameter that describes the breadth of the distribution of the rise time. For example, $\beta=1$ corresponds to a simple exponential rise, while smaller $\beta$ corresponds to a broader distribution and $\beta=0$ corresponds to a rise made up of a wide range of exponential functions with different rise times. Equation (5) can be used to deduce the rise time constant of the system by taking the logarithm of this equation twice, and then plotting $\ln (t)$ versus $\ln \left(-\ln \left(\frac{D(t)}{D_{0}}\right)\right)$ and a straight line can be obtained. The gradient of this straight line gives $\frac{1}{\beta}$ 
and the intercept gives $\ln (\tau)$ [13]. Application of this approach on the experimental data of $D(t)$ is shown in Fig. 6 for a representative sample of a $1.5 \mu \mathrm{m}$ DO11/ PMMA thin film. This approach was applied to all polymeric systems. Table III summarises the fitting results.

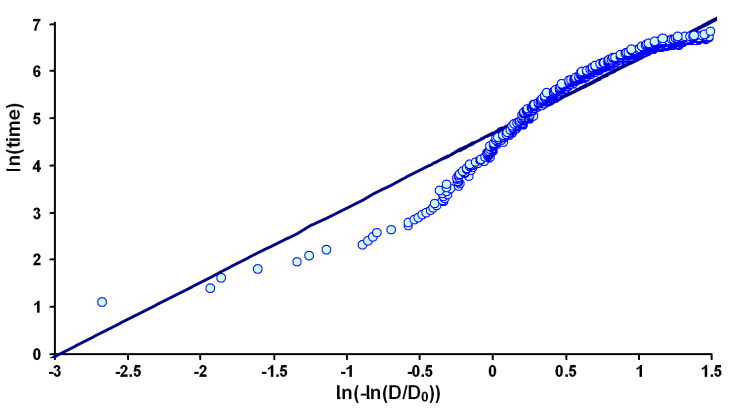

Fig. 6. A representative fit of dichroism rise to the stretched exponential function. The sample was a $1.5 \mu \mathrm{m}$ DO11/PMMA thin film.

The fact that $\beta$ is less than unity indicates that the mobility of all dye molecules is very much affected by the local environment surrounding each molecule. As such, the overall rise function can be thought of a sum of different exponential rises, which can be expressed by the stretched equation above (Eq. (5)).

\subsection{Dichroism relaxation dynamics}

Switching off the irradiation source led to a fast diminishing of dichroism, manifesting the tendency of the polymeric systems towards the isotropic state of the

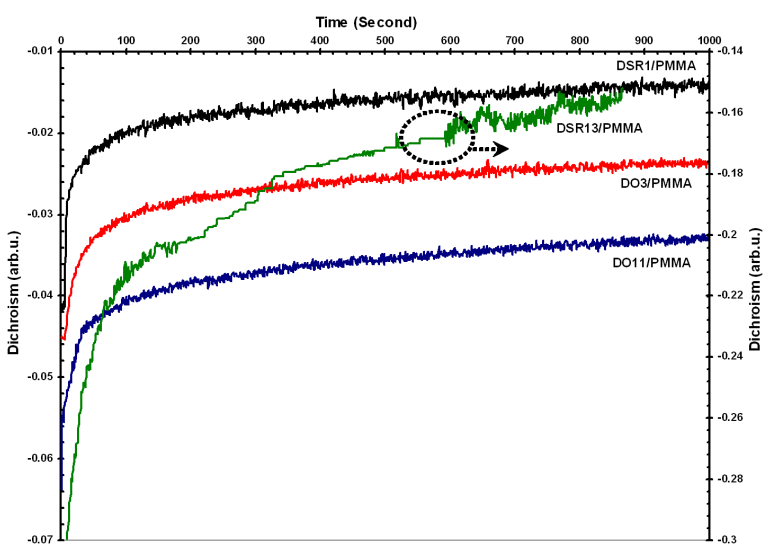

Fig. 7. Dichroism relaxation process after cutting off the polarized laser irradiation.

dye molecules distributions within the polymer networks. These observations indicate that these changes are completely reversible, leading to the conclusion that keto $\Leftrightarrow$ enol of DO11, and trans $\Leftrightarrow$ cis of other azo-besd dyes were occurring in the guest-host polymeric thin films. Figure 7 shows the photo-induced dichroism decay (relaxation) phase after cutting off the linearly polarised

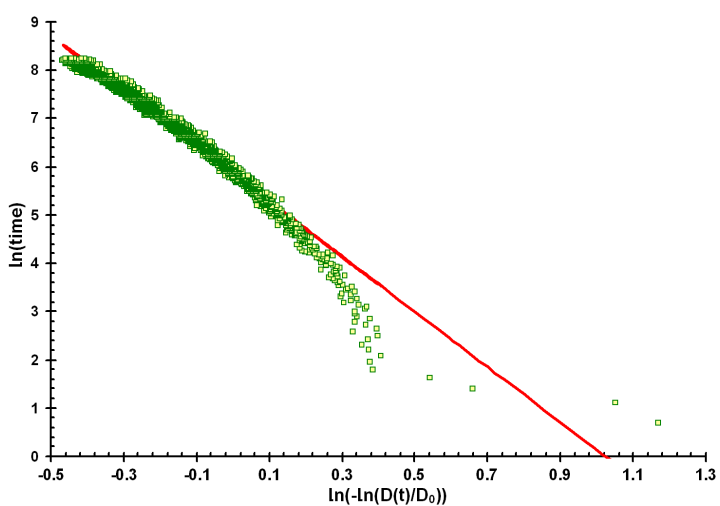

Fig. 8. A representative fit of dichroism relaxation to the stretched exponential function. The sample was a $1.5 \mu \mathrm{m}$ DO11/PMMA thin film.

irradiation source. Again, the difference in dichroism relaxation speed between those systems does reflect the difference in the molecular mobility of dye molecules within these systems. The dynamic behaviour of photo-induced dichroism decay after switching off the pump beam is fitted by Williams-Watt stretched exponential [12] of the form:

$$
D(t)=D_{o}\left[1-\exp \left(-\left(\frac{t}{\tau}\right)^{\beta}\right)\right],
$$

here, $\tau$ and $\beta$ are parameters used to describe the characteristic relaxation (decay) time and distribution breadth of the decay phase, respectively. Equation (6) can be used in a similar fashion to that used in Eq. (5) to deduce the relaxation time constant of the polymeric systems [13]. Application of this approach to the experimental data of $D(t)$ is shown in Fig. 8 for a representative $1.5 \mu \mathrm{m}$ sample of DO11/PMMA thin film. This approach was applied to all polymeric systems. Table IV summarises the fitting results.

\section{Discussion}

The results of this work emphasize the important role of the photo-induced isomerisation of azo-based organic dyes and the phototautomerisation process of DO11 dye. The role of the two processes is manifested in the polar alignment of dye molecules in the polymeric guest/host systems, which is responsible for the development of the dichroism signals in the samples $[1-2,9]$. However, the extent of dichroism signals is governed by the polar nature of the dyes as well as their volumes. Table III shows that the rising time constants of dichroism signals are in the order DSR $1<$ DSR13 < DO3 < DO11. Table V shows the dipole moments of these dyes found in literature. However, no published values related to the dipole moment of DO11 molecules have been found in the literature. For this reason, Spartan '08 (version 1.2.0) package has been used to calculate it. The calculation was performed using a restricted Hartree-Fock SCF method (basis set: $6-31 \mathrm{G}^{*}$, frequency calculation). Dipole moments 
of the rest of the dyes were also calculated under similar conditions, so that the calculation procedure was validated. As such, the value of the dipole moment of DO11 molecule was satisfactory. The calculated dipole moments are listed in Table V. Dipole moment values can be arranged in the fashion DSR13 > DSR1 > DO3 > DO11. Comparison of this result with the rising time constants of the dyes relevant dichroism values shows that polar dyes respond faster to the laser irradiation and contribute to fast polar arrangement within the polymeric structure. Nevertheless, the elevated value of $\tau$ for DO3 molecules compared to that of DO11 molecules could be attributed to the difference in the mechanisms of photoisomerisation and phototautomerisation processes. DO3 molecules isomerise under the influence of the laser radiation via the photoisomerisation route. DO11 molecules tautomerise under the influence of the laser radiation via the phototautomerisation route, in which the inter or intramolecular transfer of hydrogen (a proton) in photoexcited DO11 molecules takes place (Fig. 9). This is due to the newly formed charge density distribution of the photoexcited DO11 molecule which favours the transfer of a proton. The transferred proton normally binds to an oxygen atom yielding a $\left(\mathrm{OH}^{-}\right)$group [14]. Based on the DO11 molecular structure that is shown in Fig. 9. It seems reasonable that a hydrogen could readily bind to one of the available lone oxygens. This form is more chemically stable than the molecules without hydrogen bonds (keto type). Upon the absorption of light energy, the dye structure changes from a stable keto form in the ground state to a less stable enol form in the excited state. This enol form will mostly give off its absorbed energy rapidly and return to the keto form [14]. Furthermore, PMMA matrix can be considered as a Lewis base [15-16], so that the keto form of DO11 is likely to be very much present in the guest-host polymeric system. However, the actual volume of either forms (keto and enol), does not change much, which may explain the long rising time taken to reach the photostationary state, along with its small value of dipole moment.

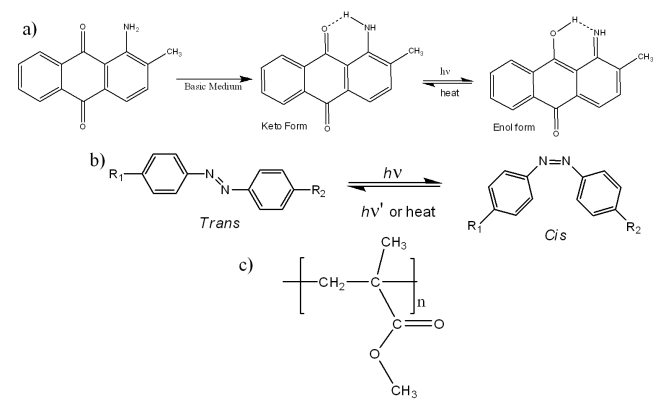

Fig. 9. (a) Phototautomerisation of DO11, (b) Photoisomerisation of Azo-dyes (c) the chemical structure of PMMA polymer.

On the other hand, an azo-based molecule in the trans isomerisation state has a bigger volume and a larger

\section{TABLE V}

Dipole moments of dyes under study.

\begin{tabular}{c|c|c|c|c}
\hline \hline & DO11 & DO3 & DSR13 & DSR1 \\
\hline $\begin{array}{c}\text { Dipole Moment* } \\
\text { [Debye] }\end{array}$ & - & 8.8 & 9.3 & 9.1 \\
\hline $\begin{array}{c}\text { Calculated Dipole } \\
\text { Moment [Debye] }\end{array}$ & 1.22 & 8.33 & 9.64 & 9.15 \\
\hline
\end{tabular}

${ }^{*}$ Ref.: [17].

dipole moment. Pumping the trans isomer with an appropriate laser wavelength drives it to change its shape into a new isomerisation shape called cis isomer (Fig. 9), which has a smaller volume and weaker dipole moment $[1-3]$. Moreover, $\beta$ values can be regarded as an indication of the volume of the dye molecules. These values are arranged in an increasing fashion such that DO3 < DSR1 < DSR13 < DO11, which illustrates further the effect of the dye molecule volume on the growth of the polar order inside the samples on one hand, and the effectiveness of the photoisomerisation and phototautomerisation processes on the other hand. For example, DO3 molecules has the smallest volume among the dyes and the smallest $\beta$, but the longest rising time constant, which indicate a poor polar alignment and less effective photoisomerisation. This could be attributed - in part - to the large free volume available for DO3 molecules in within the polymer structure. In such case, dye molecules are more likely to have their dipole moment direction aligned along the polarization direction of the linearly polarized pump beam. Furthermore, the possibility to interact with this light should not be ignored in these cases where the dipole moment direction of the dye is not perpendicular to the polarization direction of the linearly polarized pump laser light. Moreover, the very small value of $\beta$ in the case of DO3 molecules indicates that the overall rise function can be thought of an almost a single exponential rise. As for DSR13 and DSR1 dyes, the dipole moments play a clearer role in the polar order build-up process through the photoisomerisation mechanism. One can conclude that the high value of dichroism in DSR13/ PMMA samples manifests the effect of higher dipole moment of DSR 13 compared to that of DSR1. Nevertheless, the overall rise functions of both guest/host systems can be thought of a sum of different exponential rises as $\beta$ values reveal in Table III. DO11/PMMA system has a second long rising time constant as well as the largest value of $\beta$. This could be attributed to the large volume of DO11 molecule, and the overall rise function can be thought of a sum of more exponential rises. Similar behaviours are noticed in the dichroism thermal relaxation processes (Fig. 8). The decay time constants increasing in the order: DSR $1<$ DSR $13<$ DO $3<$ DO11. Both the dipole moment and the molecule volume seem to play together the essential role in the speed of the relaxation process, which is evident in the fast relaxation of DSR1/PMMA system compared to DSR13/PMMA sys- 
tem whose dipole moment is larger as well its volume due to the presence of the $\mathrm{Cl}^{-}$ion. Moreover, despite the smaller volume of DO3 molecules, its small value of dipole moment slows the relaxation process (the thermal move from the polar alignment fashion to the isotropic structure). On the other hand, $\beta$ values decrease according to DSR13 $>$ DSR1 $>$ DO11 $>$ DO3. These observations could be attributed to the geometrical volume of the dye molecules, without ignoring their dipole moment values.

The emphasis on the role of both dipole moment strength and the geometrical structure of the organic dyes (in the ground-state) in the extent of the polar alignment during irradiation should not hinder the fact, that there is another equally important factor that influences this dynamic process, namely, the excited state of the dye molecules. This can be explained by the fact that when the pump beam wavelength is at the maximum absorption peak of the dye molecules, both groundand excited-state dynamics contribute to the optically induced dichroism signal [17].

\section{Conclusions}

In this study, the dichroism characteristics of PMMA thin films doped with DO11 dye molecules under illumination with visible laser light were investigated. In addition, DO3, DSR13, and DSR1 azobenzene-based dye molecules were studied under similar experimental conditions as well. It was shown that photoisomerization and phototautomerization reactions take place in the excited state of the dye molecules, and they are consequences of the light illumination of the samples. These photo-induced processes are responsible for the optical alignment of the dyes molecules. It was shown that both photoisomerization and phototautomerization reactions are dependent on the chemical structure of the polymer as well as the photoactive dyes. Similar behaviour of dichroism growth and relaxation was observed in azo-based and anthraquinone-based guest/host polymeric systems. However, dipole moment strength and the geometrical size of dye molecules played an overwhelming role in the speed of polar order buildup in the samples as well as in the relaxation characteristics after cutting-off the irradiation laser source. PMMA/dye guest-host polymeric systems tend to relax to their stable thermodynamic state as soon as the pump light is removed.

\section{Acknowledgments}

The authors would like to express their thanks to the Director General of AECS Prof. I. Othman for his continuous encouragement, guidance and support.

\section{References}

[1] Z. Sekkat, M. Dumont, Appl. Phys. B 54, 486 (1992).

[2] M. Ivanov, L. Nikolova, T. Todorov, N. Tomova, V. Dragostinova, Opt. Quantum Electron. 26, 1013 (1994).

[3] Z. Sekkat, P. Prêtre, A. Knoesen, W. Volksen, V.Y. Lee, R.D. Miller, J. Wood, W. Knoll, J. Opt. Soc. Am. B 15, 401 (1998).

[4] I.G. Marino, R. Raschella, P.P. Lottici, D. Bersani, C. Razzetti, A. Lorenzi, A. Montenero, J. Sol-Gel Sci. Techn. 37, 201 (2006).

[5] C. Chun, E. Seo, M.J. Kim, Y.D. Shin, J.S. Lee, D.Y. Kim, Opt. Mater. 29, 970 (2007).

[6] B.F. Howell, M.G. Kuzyk, J. Opt. Soc. Am. B 19, 1790 (2002).

[7] B.F. Howell, M.G. Kuzyk, Appl. Phys. Lett. 85, 1901 (2004).

[8] K. Yamaoka, E. Charney, J. Amer. Chem. Soc. 94, 8963 (1972).

[9] B. Abbas, M. Alshikh, Khalil, Acta Phys. Pol. A 115, 857 (2009).

[10] T. Watanabe, J.L. White, Colloid Polym Sci. 270, 519 (1992).

[11] T. Kobay, T. Kobayashi, in: Photoreactive Organic Thin Films, Ed. Z. Sekkat, W. Knoll, Academic Press, San Diego 2002, p. 49.

[12] G. Williams, D.C. Watts, Trans. Faraday Soc. 66, 80 (1970).

[13] K.D. Singer, L.A. King, J. Appl. Phys. 70, 3251 (1991).

[14] W. Chu, S.M. Tsui, J. Environ. Eng. 127, 741 (2001).

[15] F.M. Fowkes, D.O. Tischler, J.A. Wolfe, L.A. Lannigan, C.M. Ademu-John, M.J. Halliwell, J. Polym. Sci., Chem. Ed. 22, 547 (1984).

[16] N. Shahidzadeh, M.M. Chehimi, F. Arefi-Khonsari, J. Amouroux, M. Delamar, Plasma Polym. 1, 27 (1996).

[17] S. Mukamel, Principles of Nonlinear Optical Spectroscopy, Oxford University Press, New York 1995, p. 454 . 